\title{
Allogeneic Nicotinamide-expanded Natural Killer Cells
}

National Cancer Institute

\section{Source}

National Cancer Institute. Allogeneic Nicotinamide-expanded Natural Killer Cells. NCI

Thesaurus. Code C143157.

Allogeneic, nicotinamide (NAM)-expanded natural killer (NK) cells, with potential cytolytic and antineoplastic activities. Upon administration, the allogeneic NAM-expanded NK cells may lyse cancer cells. These cells also secrete pro-inflammatory cytokines, which further stimulate an anti-tumor immune response. Ex-vivo treatment with the vitamin B3 derivative NAM increases the in-vivo homing, retention and proliferation potential of the NK cells. 